\title{
Direct Medical Costs of 3 Reportable Travel-Related Infections in Ontario, Canada, 2012-2014
}

\author{
Rachel D. Savage, Laura C. Rosella, Natasha S. Crowcroft, \\ Maureen Horn, Kamran Khan, Laura Holder, Monali Varia
}

Immigrants traveling to their birth countries to visit friends or relatives are disproportionately affected by travel-related infections, in part because most preventive travel health services are not publicly funded. To help identify cost-effective policies to reduce this disparity, we measured the medical costs (in 2015 Canadian dollars) of 3 reportable travel-related infectious diseases (hepatitis A, malaria, and enteric fever) that accrued during a 3-year period (2012-2014) in an ethnoculturally diverse region of Canada (Peel, Ontario) by linking reportable disease surveillance and health administrative data. In total, 318 case-patients were included, each matched with 2 controls. Most spending accrued in inpatient settings. Direct healthcare spending totaled $\$ 2,058,196$; the mean attributable cost per case was $\$ 6,098(95 \% \mathrm{Cl}$ $\$ 5,328-\$ 6,868$ ) but varied by disease (range $\$ 4,558-$ $\$ 7,852)$. Costs were greatest for enteric fever. Policies that address financial barriers to preventive health services for high-risk groups should be evaluated.

$\mathrm{B}$ ecause of the rapid growth of air travel and immigration, more travelers worldwide are exposed to nonendemic infectious diseases (e.g., Zika, measles, malaria) than ever before (1-3). In Ontario, Canada, >3,000 travelrelated infections are reported to public health annually (4); this number is an underestimate because not all sick persons seek healthcare treatment, especially while traveling, and not all conditions are diagnosed and reported. Immigrant travelers who return to their birth countries to visit friends or relatives are a substantial risk group (5). In Canada and elsewhere, regions with high proportions of immigrant travelers to South Asia and Africa have the highest

Author affiliations: Women's College Hospital, Toronto, Ontario, Canada (R.D. Savage); University of Toronto, Toronto

(R.D. Savage, L.C. Rosella, N.S. Crowcroft, K. Khan); ICES,

Toronto (R.D. Savage, L.C. Rosella, L. Holder); Public Health

Ontario, Toronto (N.S. Crowcroft); Peel Public Health,

Mississauga, Ontario, Canada (M. Horn, M. Varia); St. Michael's

Hospital, Toronto (K. Khan)

DOI: https://doi.org/10.3201/eid2508.190222 rates of imported cases of hepatitis $\mathrm{A}$, malaria, and enteric fever (4,6-8). The disproportionate burden of travel-related infections in immigrants has been attributed to their traveling to riskier destinations (9) and prolonged travel stays $(10,11)$ but also to their poor uptake of pretravel health services $(10,12-14)$.

Pretravel health consultations provide an opportunity to intervene and reduce travel-related infections (14). The Committee to Advise on Tropical Medicine and Travel recommends that nonimmune travelers going to developing countries receive the hepatitis A vaccine (15), travelers going to South Asia receive the typhoid vaccine (16), and travelers going to regions where malaria is endemic receive chemoprophylaxis (17) before traveling. Despite these recommendations, pretravel health services are generally not covered by provincial universal insurance plans, with few exceptions (18). Private health insurance can fill these gaps by providing partial or complete coverage for these services; however, many travelers, including those visiting friends or relatives (VFR), who are at greater risk for infection, often do not have private insurance. The cost of pretravel health services has been described by VFR travelers as a barrier $(9,19-22)$. As a result, public health officials have advocated for universal coverage of pretravel health services to reduce the substantial public health resources required for the management of these imported cases $(6,23)$.

The direct medical costs of reportable travel-related infections to healthcare systems has not been measured. The existing estimates were determined primarily by using inpatient settings or are considered outdated (24-26). As outbound travel and annual immigration targets continue to increase, evidence is needed to determine if policies are meeting the healthcare needs of an increasingly diverse population. Furthermore, mathematic and economic models require this information as inputs, so the lack of cost estimates has limited the development of these models. In this report, we sought to measure healthcare utilization and attributable medical costs of 3 key reportable travel-related 
infections in an ethnoculturally diverse region of Canada by linking public health reportable disease surveillance data with health administrative data.

\section{Materials and Methods}

\section{Design and Setting}

We received ethics approval (no. 31366) for this study from the University of Toronto Research Ethics Board (Toronto, Canada). We used a population-based, matched-cohort design to estimate attributable medical costs of incident cases of hepatitis A, malaria, and enteric fever from a healthcare payer perspective. This study was conducted in the Peel region of Ontario, one of Canada's largest and most ethnoculturally diverse municipalities, which has $\approx 1.4$ million residents, $\approx 50 \%$ of whom are foreign-born (27). South Asians are the largest visible minority in both the Peel region and Canada (28). For the purposes of our study, we needed to link reportable disease surveillance data with the health administrative data collected as part of Ontario's government-funded, universal healthcare. Each data source independently would have been insufficient to achieve the study objective, and thus, the linkage represents a valuable feature of this study.

\section{Case-Patients}

During the study period, reporting hepatitis A, malaria, and enteric fever (i.e., typhoid fever and paratyphoid fever) to public health authorities in Ontario was required by the Health Protection and Promotion Act (29). We identified the laboratory-confirmed hepatitis A, malaria, and enteric fever case-patients reported to Peel Public Health during January 1, 2012-December 31, 2014, using the integrated Public Health Information System (iPHIS). We excluded case-patients who were unable to be linked to the Ontario Registered Persons Database, which contains the demographic information of all persons issued an Ontario health card, and we compared linked and unlinked case-patients to identify potential sources of bias (30). Because reporting is known to be incomplete, we additionally identified Peel region residents with information in the health administrative datasets who were hospitalized with the following diagnostic codes from the International Classification of Diseases, 10th Revision, with Canadian Enhancements: hepatitis A (B15), malaria (B50-54), or typhoid and paratyphoid fever (A01). If hospitalizations were recurrent, we counted the series once (i.e., we considered all hospitalizations to be a part of the same case). We excluded case-patients identified in the health administrative datasets if their healthcare record indicated uncertainty of the diagnosis (31). Index dates were based on the iPHIS episode accurate date (i.e., the symptom onset date for $95 \%$ of case-patients and specimen collection date for the remainder) or the case-patient's hospital admission date. Index dates for iPHIS case-patients were December 20, 2011-December 17, 2014. To account for delays between symptom onset and healthcare seeking, we included case-patients from hospitalization records if they were admitted during December 20, 2011-January 4, 2015 . We chose to add this $\approx 3$-week extension to the end of the accrual window because iPHIS case-patients had, on average, an 18-day delay between symptom onset and healthcare presentation.

\section{Controls}

For each disease, we matched 2 controls per case-patient from a pool of eligible controls in the health administrative datasets. We used controls to determine the baseline medical costs so that we could calculate the costs attributable to travel. Controls were eligible if they were registrants of the Ontario Health Insurance Program, had contact with the healthcare system within the 3 years before their assigned index date, and resided in the Peel region (according to their postal code) during the period of study (Figure 1). We excluded persons from the pool of eligible controls if they had a travel-related diagnostic code during the study period (Table 1). We randomly assigned index dates to eligible controls according to the index date distribution of casepatients and then excluded controls who did not reside in the Peel region or had died as of their assigned index date.

\section{Matching and Covariates}

For each disease, we matched controls to case-patients by index date ( \pm 60 days), age ( \pm 3 years), sex, neighborhood income quintile, foreign-born status, years in Canada if foreign-born $(0-4,5-9$, or $\geq 10)$, and concurrent medical conditions. We estimated neighborhood income quintile by linking case-patients' postal codes to existing average household income data for their neighborhoods and stratifying neighborhoods by quintile to generate neighborhood-specific income quintiles (32). We enhanced the study cohort by linking to the Ontario portion of the Immigration, Refugees, and Citizenship Canada Permanent Resident (IRCC-PR) database (1985-2012) (33). We defined case-patients as foreign-born if they had a country of birth outside of Canada recorded in iPHIS or if they had a record present in the IRCC-PR database. We designated controls as foreign-born solely using IRCC-PR data. To calculate years in Canada, we used the immigration date in the IRCC-PR database. For immigrants not yet captured in the IRCC-PR database (i.e., immigrants who landed in Ontario after 2012), we assigned their immigration date as the date 3 months before their first healthcare contact because new Ontario residents have a 3-month waiting period before they become eligible for the Ontario Health Insurance Program (34). For a small number of case-patients $(n<6)$, we set their years in Canada to 0 because their index date 


\section{$\mathbf{1 4 , 4 7 6 , 0 6 4}$ persons in Ontario born before accrual window (December 20, 2011-December 17, 2014), who had a date of last healthcare contact $<3$ years before accrual start date \\ $13,426,901$ non-Peel residents during 2011-2014}

\begin{tabular}{|l|l|}
\hline $\mathbf{1 , 0 4 9 , 1 6 3}$ persons \\
$\qquad \begin{array}{l}337,931 \text { with a travel-related } \\
\text { diagnostic code during } \\
\text { accrual window }\end{array}$
\end{tabular}

\section{1,232 persons*}

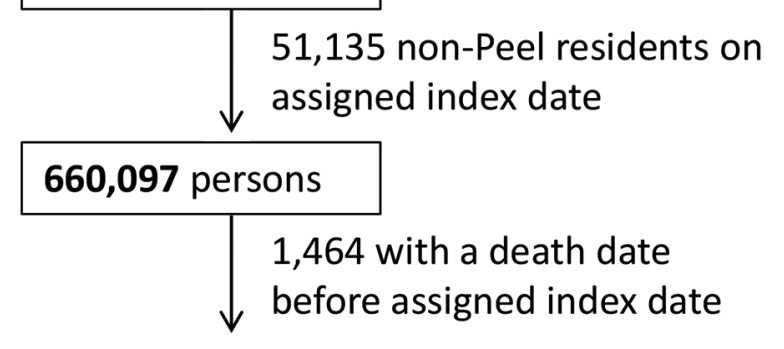

658,633 persons eligible as controls

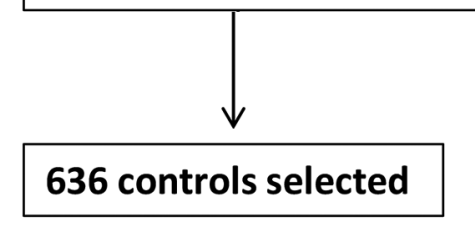

Figure 1. Flowchart of control selection in study of direct costs of hepatitis A, malaria, and enteric fever, Peel region, Ontario, Canada, 2011-2014. *Index date randomly assigned on the basis of the index date distribution of case-patients.

preceded their first contact with healthcare. We determined the concurrent medical conditions of case-patients and controls during the 2 years before their index date by using the Johns Hopkins ACG System collapsed Aggregated Diagnostic Groups validated for use in Ontario $(35,36)$. We further collapsed categories of like duration and severity of condition (i.e., unstable chronic conditions requiring medical [category 5] or specialty care [category 9], stable chronic conditions requiring medical [category 6] or specialty care [category 7]).

\section{Healthcare Utilization and Costs}

We measured healthcare utilization and the medical costs that occurred up to 90 days after and including the index date; we chose this period on the basis of published
Table 1. Diagnostic codes used to identify and exclude noneligible controls in study of direct costs of hepatitis A, malaria, and enteric fever, Peel region, Ontario, Canada, 2012-2014*

\begin{tabular}{|c|c|c|}
\hline Disease & ICD-10-CA & OHIP \\
\hline Hepatitis A & $\begin{array}{c}\text { B15, B19, A09, A08.3- } \\
\text { A08.5 }\end{array}$ & $\begin{array}{c}\text { 070, 009, 079, 136, } \\
787\end{array}$ \\
\hline Malaria & $\begin{array}{c}\text { B50-B54, P37.3-P37.4, } \\
\text { B64 }\end{array}$ & $\begin{array}{c}062,136,781,784 \\
787\end{array}$ \\
\hline Enteric fever & $\begin{array}{c}\mathrm{A} 01, \mathrm{~A} 02.9, \mathrm{~A} 02.1, \mathrm{~A} 04.9 \\
\mathrm{~A} 05.9, \mathrm{~A} 09, \mathrm{~A} 49.9\end{array}$ & $\begin{array}{c}002,009,003,005 \\
136,784,787\end{array}$ \\
\hline
\end{tabular}

estimates of illness duration (37). We probabilistically linked case-patients present in iPHIS to their health administrative data with unique identifiers and used a validated person-centered costing method developed for analyzing Ontario health administrative data to estimate direct medical costs (38). Those datasets were linked using unique encoded identifiers and analyzed at ICES (Toronto, Canada). The costing method used captured all relevant costs covered by the Ontario single-payer health insurance plan, including inpatient hospitalizations, emergency department (ED) visits, same-day surgery, dialysis, oncology clinic visits, feefor-service physician and nonphysician services, non-feefor-service physicians, prescription medications, laboratory services, rehabilitation, complex continuing care, long-term care, mental health inpatient stays, and home care services. We adjusted costs for inflation by using health sector-specific consumer price indices and reported all costs in 2015 Canadian dollars (Can).

\section{Analysis}

We assessed the quality of matching by comparing case-patients and controls using the standardized difference $(d)$; we used a $d$ value of $>0.10$ to identify significant imbalances $(39,40)$. We estimated attributable, 90 -day per-person costs as the mean of the differences in costs among case-patientcontrol pairs (41). To account for the clustered nature of the data (i.e., 2 controls per case-patient), we used generalized estimating equations with an exchangeable correlation matrix to estimate $95 \%$ CIs (41). We stratified all attributable cost estimates by disease and setting and, for malaria, by infecting species. We categorized inpatient settings as inpatient hospitalizations, inpatient mental health hospitalizations, rehabilitation services, and complex continuing care; ED settings as ED visits and shadow billings to ED physicians; and the remainder of settings as outpatient settings. We performed analyses using SAS version 9.3 (https://www.sas.com).

\section{Sensitivity Analyses}

To examine the effect of healthcare database selection and the time window on estimated costs, we repeated our analysis in 2 ways. In the first sensitivity analysis, we included 
only the cost calculations for expenditures considered most relevant to diagnosing and treating acute infections (i.e., inpatient hospitalizations, ED visits, same-day surgery, fee-for-service physician and nonphysician services, nonfee-for-service physicians, prescription medications, laboratory services). In the second, we limited the measurement of costs to a 60-day follow-up period.

\section{Results}

During 2012-2014, a total of 289 cases of hepatitis A, malaria, or enteric fever were reported to Peel Public Health in case-patients linkable to the health administrative data, a linkage ratio of $90 \%(289 / 321)$. No deaths were identified among the case-patients. Unlinked case-patients $(\mathrm{n}=32)$ were more likely than linked case-patients $(n=289)$ to be recent immigrants (i.e., immigrants who arrived in Ontario during the incubation period of their disease) or visitors (53.3\% unlinked vs. $8.7 \%$ linked; $p<0.001)$, but otherwise the linked and unlinked case-patients were similar in terms of sex, age, disease distribution, and foreign-born status. We identified an additional 29 case-patients in hospital records for a total case-patient cohort size of 318 .

Compared with all (unmatched) eligible controls, casepatients were younger and a higher proportion were male (Table 2). Case-patients were also more likely than unmatched controls to be foreign-born, have acute or stable chronic medical conditions, and live in neighborhoods of lower income quintiles. Case-patients and matched controls were similar in terms of all matching variables, with the exception of missing neighborhood income quintile (Tables 2, 3).

Table 2. Characteristics of case-patients in study of direct costs of hepatitis A, malaria, and enteric fever and pools of unmatched and matched eligible controls, Peel region, Ontario, Canada, 2012-2014*

\begin{tabular}{|c|c|c|c|c|c|}
\hline \multirow[b]{2}{*}{ Variable } & \multirow[b]{2}{*}{ Case-patients, $\mathrm{n}=318$} & \multicolumn{2}{|c|}{ Prematching } & \multicolumn{2}{|c|}{ Postmatching } \\
\hline & & Controls, $n=658,633$ & $d$ & Controls, $n=636$ & $d$ \\
\hline \multicolumn{6}{|l|}{ Age, y } \\
\hline Mean \pm SD & $31 \pm 21$ & $37 \pm 22$ & 0.273 & $31 \pm 21$ & 0.001 \\
\hline Median (IQR) & $30(12-47)$ & $36(20-53)$ & 0.268 & $30(12-47)$ & 0.001 \\
\hline$<1$ & 0 & $9,694(1.5)$ & 0.173 & 0 & 0 \\
\hline $1-4$ & $21(6.6)$ & $27,381(4.2)$ & 0.109 & $42(6.6)$ & 0 \\
\hline $5-9$ & $36(11.3)$ & $40,692(6.2)$ & 0.183 & 75 (11.8) & 0.015 \\
\hline $10-14$ & $33(10.4)$ & 41,235 (6.3) & 0.149 & $63(9.9)$ & 0.016 \\
\hline $15-24$ & $43(13.5)$ & $93,870(14.3)$ & 0.021 & $85(13.4)$ & 0.005 \\
\hline $25-49$ & $120(37.7)$ & $245,090(37.2)$ & 0.011 & $240(37.7)$ & 0 \\
\hline $50-74$ & 57 (17.9) & $167,999(25.5)$ & 0.185 & 115 (18.1) & 0.004 \\
\hline$\geq 75$ & $8(2.5)$ & $32,672(5.0)$ & 0.129 & $16(2.5)$ & 0 \\
\hline \multicolumn{6}{|l|}{ Sex } \\
\hline $\mathrm{F}$ & $123(38.7)$ & $321,510(48.8)$ & 0.205 & $252(39.6)$ & 0.019 \\
\hline $\mathrm{M}$ & $195(61.3)$ & $337,123(51.2)$ & 0.205 & $384(60.4)$ & 0.019 \\
\hline \multicolumn{6}{|c|}{ Neighborhood income quintile } \\
\hline 1 , lowest & $70(22.0)$ & $103,751(15.8)$ & 0.160 & $133(20.9)$ & 0.027 \\
\hline 2 & $88(27.7)$ & $109,728(16.7)$ & 0.268 & 169 (26.6) & 0.025 \\
\hline 3 & $94(29.6)$ & $121,601(18.5)$ & 0.262 & $203(31.9)$ & 0.051 \\
\hline 4 or 5 , highest & $60-70(\mathrm{~S})$ & $159,348(24.2)$ & 0.231 & $131(20.6)$ & 0.027 \\
\hline Missing & $<6(\mathrm{~S})$ & $356(0.1)$ & 0.150 & 0 & 0.160 \\
\hline \multicolumn{6}{|l|}{ Foreign-born, database } \\
\hline IRCC-PR† & $183(57.5)$ & $73,564(11.2)$ & 1.119 & 449 (70.6) & 0.275 \\
\hline iPHIS or IRCC-PR & $227(71.4)$ & $73,564(11.2)$ & 1.546 & 449 (70.6) & 0.017 \\
\hline \multicolumn{6}{|c|}{ Years in Canada, no./total (\%) } \\
\hline $0-4$ & $58 / 227(25.6)$ & $13,535 / 73,564(18.4)$ & 0.170 & $113 / 449(25.2)$ & 0.009 \\
\hline $5-9$ & $56 / 227(24.7)$ & $15,868 / 73,564(21.6)$ & 0.070 & $114 / 449(25.4)$ & 0.017 \\
\hline$\geq 10$ & $113 / 227(49.8)$ & $44,161 / 73,564(60.0)$ & 0.210 & $222 / 449(49.4)$ & 0.007 \\
\hline \multicolumn{6}{|c|}{ Concurrent medical condition§ } \\
\hline 1 & $240(75.5)$ & 351,379 (53.3) & 0.475 & $475(74.7)$ & 0.018 \\
\hline 2 & $210(66.0)$ & 313,777 (47.6) & 0.378 & $420(66.0)$ & 0 \\
\hline 3 & $178(56.0)$ & $286,375(43.5)$ & 0.252 & $359(56.4)$ & 0.010 \\
\hline 4 & $28(8.8)$ & $33,259(5.0)$ & 0.148 & $59(9.3)$ & 0.016 \\
\hline 5 and 9 & $44(13.8)$ & $90,116(13.7)$ & 0.004 & $118(18.6)$ & 0.008 \\
\hline 6 and 7 & 107 (33.6) & $164,535(25.0)$ & 0.191 & $222(34.9)$ & 0.007 \\
\hline 8 & $12(3.8)$ & $39,357(6.0)$ & 0.102 & $23(3.6)$ & 0.008 \\
\hline 10 & 63 (19.8) & $135,170(20.5)$ & 0.018 & $127(20.0)$ & 0.004 \\
\hline 11 & $119(37.4)$ & 216,829 (32.9) & 0.094 & $216(34.0)$ & 0.072 \\
\hline 12 & $12(3.8)$ & $15,640(2.4)$ & 0.081 & 24 (3.8) & 0 \\
\hline
\end{tabular}

*Values are no. (\%) except as indicated. $d$, standardized difference; iPHIS, integrated Public Health Information System; IQR, interquartile range; IRCC-PR, Immigration, Refugee and Citizenship Canada Permanent Resident; S, suppressed per ICES reidentification risk assessment policy. †Case-patients and controls were designated as foreign-born if they had a record in the IRCC-PR database.

¥Case-patients were designated as foreign-born if country of birth was listed as outside of Canada in iPHIS, or they had a record in the IRCC-PR database; controls were designated as foreign-born if they had a record in the IRCC-PR database.

§Listed by John Hopkins collapsed Aggregated Diagnostic Group: 1 (acute minor), 2 (acute major), 3 (likely to recur), 4 (asthma), 5 and 9 (chronic unstable), 6 and 7 (chronic stable), 8 (eye and dental), 10 (psychosocial), 11 (preventive and administrative), and 12 (pregnancy). 
Most (>90\%) case-patients had travel-associated illnesses (Table 4); of these case-patients, most (50\%) traveled to India, and more than half $(63 \%)$ reported traveling to visit friends or relatives. A similar proportion of children $<16$ years of age (57\% [43/76]) also reported traveling to visit friends or relatives, although this proportion varied by disease $(47 \%$ [8/17] for hepatitis A, 40\% [6/15] for malaria, 66\% [29/44] for enteric fever). Most of the case-patients identified as recent immigrants received malaria diagnoses, and of these, 65\% (13/20) were caused by Plasmodium vivax, probably representing relapsed malaria rather than primary disease acquired by travel. Casepatients with $P$. falciparum malaria primarily traveled to West Africa $(83 \%, 40 / 48)$, and all case-patients with $P$. vivax malaria had traveled to India $(58 \%, 15 / 26)$ or
Pakistan (42\%, 11/26). Few case-patients reported having a pretravel health consultation.

Of the 318 case-patients, 197 (61.9\%) were hospitalized, $232(73.0 \%)$ visited the ED, and 298 (93.7\%) visited a physician (i.e., family or general practice physician or specialist) for their illness. However, a total of 225 hospitalizations, 429 ED visits, and 4,831 unique physician visits occurred (Table 5); more than half of these encounters were for enteric fever diagnoses or treatment.

The overall cost of the 318 travel-related infections that occurred during the 3-year study period in Peel was $\$ 2,058,196$ (Table 6). Extrapolating the estimated mean cost of these infections per case-patient for the Peel region to the Ontario case counts (42) amounted to a total of $\$ 7,870,341$ in direct healthcare spending. More than one

Table 3. Characteristics of case-patients and matched controls in study of direct costs of hepatitis A, malaria, and enteric fever, by disease, Peel region, Ontario, Canada, 2012-2014*

\begin{tabular}{|c|c|c|c|c|c|c|c|c|c|}
\hline \multirow[b]{2}{*}{ Variable } & \multicolumn{3}{|c|}{ Hepatitis A } & \multicolumn{3}{|c|}{ Malaria } & \multicolumn{3}{|c|}{ Enteric fever } \\
\hline & $\begin{array}{c}\text { Case-patients, } \\
n=55\end{array}$ & $\begin{array}{c}\text { Controls, } \\
n=110\end{array}$ & $d$ & $\begin{array}{c}\text { Case- } \\
\text { patients, } \\
\mathrm{n}=122\end{array}$ & $\begin{array}{c}\text { Controls, } \\
n=244\end{array}$ & $d$ & $\begin{array}{c}\text { Case- } \\
\text { patients, } \\
\mathrm{n}=141\end{array}$ & $\begin{array}{c}\text { Controls, } \\
n=282\end{array}$ & $d$ \\
\hline \multicolumn{10}{|l|}{ Age, y } \\
\hline Median \pm IQR & $24 \pm 21$ & $24 \pm 21$ & 0 & $40 \pm 19$ & $40 \pm 19$ & 0.001 & $26 \pm 19$ & $26 \pm 19$ & 0.002 \\
\hline Mean (SD) & $19(10-32)$ & $19(10-32)$ & 0.003 & $43(27-55)$ & $43(27-55)$ & 0.002 & $25(10-40)$ & $25(9-40)$ & 0.001 \\
\hline $0-9$ & $13(23.6)$ & $23(23.6)$ & 0 & $10(8.2)$ & $20(8.2)$ & 0 & $34(24.1)$ & $71(25.1)$ & 0.025 \\
\hline $10-14$ & 7 (12.7) & $14(12.7)$ & 0 & $7(5.7)$ & $14(5.7)$ & 0 & 19 (13.5) & $35(12.4)$ & 0.032 \\
\hline $15-24$ & $17(30.9)$ & 34 (30.9) & 0 & $10(8.2)$ & $20(8.2)$ & 0 & $16(11.3)$ & $31(11.0)$ & 0.011 \\
\hline $25-49$ & $12(21.8)$ & $24(21.8)$ & 0 & 54 (44.3) & 107 (43.9) & 0.008 & 54 (38.3) & 109 (38.7) & 0.007 \\
\hline$\geq 50$ & $6(11.0)$ & $12(11.0)$ & 0 & $41(33.6)$ & $83(34.0)$ & 0.008 & $18(12.8)$ & $36(12.8)$ & 0 \\
\hline \multicolumn{10}{|l|}{ Sex } \\
\hline $\mathrm{F}$ & $27(49.1)$ & $54(49.1)$ & 0 & 37 (30.3) & $78(32.0)$ & 0.035 & $59(41.8)$ & $120(42.6)$ & 0.014 \\
\hline $\mathrm{M}$ & $28(50.9)$ & $56(50.9)$ & 0 & $85(69.7)$ & $166(68.0)$ & 0.035 & $82(58.2)$ & $162(57.4)$ & 0.014 \\
\hline \multicolumn{10}{|l|}{ Income quintile } \\
\hline 1 , lowest & $9(16.4)$ & $15(13.6)$ & 0.076 & $36(29.5)$ & 68 (27.9) & 0.036 & $25(17.7)$ & $50(17.7)$ & 0 \\
\hline 2 & $6(10.9)$ & $10(9.1)$ & 0.061 & $39(32.0)$ & 77 (31.6) & 0.009 & 43 (30.5) & $82(29.1)$ & 0.031 \\
\hline 3 & $17(30.9)$ & $36(32.7)$ & 0.039 & $20-25(\mathrm{~S})$ & $56(23.0)$ & 0.060 & $52(36.9)$ & $111(39.4)$ & 0.051 \\
\hline 4 and 5 , highest & $23(41.8)$ & 49 (44.5) & 0.055 & $20-25$ (S) & 43 (17.6) & 0.033 & 15-20 (S) & $39(13.8)$ & 0.010 \\
\hline Missing & 0 & 0 & 0 & $<6(\mathrm{~S})$ & 0 & 0.183 & $<6(\mathrm{~S})$ & 0 & 0.170 \\
\hline Foreign-born & $30(54.5)$ & $58(52.7)$ & 0.036 & $101(82.8)$ & $200(82.0)$ & 0.022 & $96(68.1)$ & $191(67.7)$ & 0.008 \\
\hline \multicolumn{10}{|c|}{ Years in Canada, no./total (\%) } \\
\hline $0-4$ & $\begin{array}{c}6 / 30 \\
(20.0)\end{array}$ & $\begin{array}{l}10 / 58 \\
(17.2)\end{array}$ & 0.071 & $\begin{array}{c}29 / 101 \\
(28.7)\end{array}$ & $\begin{array}{c}63 / 200 \\
(31.5)\end{array}$ & 0.061 & $\begin{array}{l}23 / 96 \\
(24.0)\end{array}$ & $\begin{array}{c}40 / 191 \\
(20.9)\end{array}$ & 0.072 \\
\hline $5-9$ & $\begin{array}{l}10 / 30 \\
(33.3)\end{array}$ & $\begin{array}{l}20 / 58 \\
(34.5)\end{array}$ & 0.024 & $\begin{array}{c}21 / 101 \\
(20.8)\end{array}$ & $\begin{array}{c}40 / 200 \\
(20.0)\end{array}$ & 0.020 & $\begin{array}{l}25 / 96 \\
(26.0)\end{array}$ & $\begin{array}{c}54 / 191 \\
(28.3)\end{array}$ & 0.050 \\
\hline$\geq 10$ & $\begin{array}{r}14 / 30 \\
(46.7)\end{array}$ & $\begin{array}{l}28 / 58 \\
(48.3)\end{array}$ & 0.032 & $\begin{array}{c}51 / 101 \\
(50.5)\end{array}$ & $\begin{array}{c}97 / 200 \\
(48.5)\end{array}$ & 0.040 & $\begin{array}{l}48 / 96 \\
(50.0)\end{array}$ & $\begin{array}{c}97 / 191 \\
(50.8)\end{array}$ & 0.016 \\
\hline \multicolumn{10}{|c|}{ Concurrent medical conditions $†$} \\
\hline 1 & 43 (78.2) & $84(76.4)$ & 0.043 & $84(68.9)$ & $166(68.0)$ & 0.018 & $113(80.1)$ & $225(79.8)$ & 0.009 \\
\hline 2 & $37(67.3)$ & $75(68.2)$ & 0.019 & $78(63.9)$ & $161(66.0)$ & 0.043 & $95(67.4)$ & $184(65.2)$ & 0.045 \\
\hline 3 & $32(58.2)$ & $68(61.8)$ & 0.074 & $59(48.4)$ & $118(48.4)$ & 0 & $87(61.7)$ & $173(61.3)$ & 0.007 \\
\hline 4 & $<6(\mathrm{~S})$ & $6(5.5)$ & 0 & $7(5.7)$ & $17(7.0)$ & 0.050 & $18(12.8)$ & $36(12.8)$ & 0 \\
\hline 5 and 9 & $11(20.0)$ & 19 (17.3) & 0.070 & 25 (20.5) & $56(23.0)$ & 0.060 & 24 (17.0) & 43 (15.2) & 0.048 \\
\hline 6 and 7 & $13(23.6)$ & $25(22.7)$ & 0.022 & $49(40.2)$ & $98(40.2)$ & 0 & $50(35.5)$ & $99(35.1)$ & 0.007 \\
\hline 8 & $<6(\mathrm{~S})$ & $<6(\mathrm{~S})$ & 0 & $<6(\mathrm{~S})$ & $9(3.7)$ & 0.022 & $7(5.0)$ & $12(4.3)$ & 0.034 \\
\hline 10 & $12(21.8)$ & $26(23.6)$ & 0.043 & $24(19.7)$ & $44(18.0)$ & 0.042 & 27 (19.1) & $57(20.2)$ & 0.027 \\
\hline 11 & $23(41.8)$ & 42 (38.2) & 0.074 & 37 (30.3) & 69 (28.3) & 0.045 & $59(41.8)$ & 105 (37.2) & 0.094 \\
\hline 12 & $<6(\mathrm{~S})$ & $<6(\mathrm{~S})$ & 0 & $<6(\mathrm{~S})$ & $12(4.9)$ & 0.040 & $<6(\mathrm{~S})$ & $8(2.8)$ & 0.040 \\
\hline
\end{tabular}

${ }^{*}$ Values are no. (\%) except as indicated. $d$, standardized difference; IQR, interquartile range; S, suppressed per ICES reidentification risk assessment policy.

†Listed by John Hopkins collapsed Aggregated Diagnostic Group: 1 (acute minor), 2 (acute major), 3 (likely to recur), 4 (asthma), 5 and 9 (chronic unstable), 6 and 7 (chronic stable), 8 (eye and dental), 10 (psychosocial), 11 (preventive and administrative), and 12 (pregnancy). 
Table 4. Travel-related characteristics of case-patients with hepatitis A, malaria, or enteric fever reported to public health, Peel region, Ontario, Canada, 2012-2014*

\begin{tabular}{|c|c|c|c|c|}
\hline \multirow[b]{2}{*}{ Characteristic } & \multicolumn{4}{|c|}{ No. $(\%)$ case-patients } \\
\hline & Hepatitis A & Malaria & Enteric fever & Overall \\
\hline \multicolumn{5}{|l|}{ Travel associated $\dagger$} \\
\hline Yes & $39(79.6)$ & $103(91.2)$ & $120(94.5)$ & $262(90.7)$ \\
\hline No or unknown & $10(20.4)$ & $10(8.8)$ & $7(5.5)$ & $27(9.3)$ \\
\hline \multicolumn{5}{|l|}{ Region of birth $\dagger$} \\
\hline South Asia & $10(20.4)$ & $38(33.6)$ & $78(61.4)$ & $126(43.6)$ \\
\hline North America & $14(28.6)$ & $6(5.3)$ & $35(27.6)$ & $55(19.0)$ \\
\hline West Africa & 0 & $29(25.7)$ & 0 & $29(10.0)$ \\
\hline Other or missing & $25(51.0)$ & $40(35.4)$ & $14(11.0)$ & $79(27.3)$ \\
\hline \multicolumn{5}{|l|}{ Primary travel country $\ddagger$} \\
\hline India & $11(29.0)$ & $16(19.5)$ & $92(78.6)$ & $119(50.2)$ \\
\hline Pakistan & $14(36.8)$ & $11(13.4)$ & $19(16.2)$ & $44(18.6)$ \\
\hline Nigeria & 0 & $20(24.4)$ & 0 & $20(8.4)$ \\
\hline Ghana & 0 & $19(23.2)$ & 0 & $19(8.0)$ \\
\hline Other or missing & $13(34.2)$ & $16(19.5)$ & $6(5.1)$ & $35(14.8)$ \\
\hline \multicolumn{5}{|l|}{ Purpose of travel $₫ \S$} \\
\hline Visiting friends or relatives & $18(47.4)$ & $45(54.9)$ & $87(74.4)$ & $150(63.3)$ \\
\hline Leisure, business, or other & $<6(\mathrm{~S})$ & $13(15.9)$ & $11(9.4)$ & $25-29(\mathrm{~S})$ \\
\hline Missing & $17(44.7)$ & $27(32.9)$ & $24(20.5)$ & $68(28.7)$ \\
\hline \multicolumn{5}{|l|}{ Pretravel health consultation $\ddagger$} \\
\hline Yes & $<6(\mathrm{~S})$ & $14(17.1)$ & $10(8.5)$ & $25-29(\mathrm{~S})$ \\
\hline No or unknown & $33-38(S)$ & $68(82.9)$ & $107(91.5)$ & $208-213(\mathrm{~S})$ \\
\hline \multicolumn{5}{|c|}{$\begin{array}{l}\text { "S, suppressed per ICES reidentification risk assessment policy. } \\
\text { †Includes recent immigrants (i.e., immigrants who arrived in Ontario during the incubation period of their disease) and visitors: hepatitis } A(n=49) \text {, } \\
\text { malaria }(n=113) \text {, enteric fever }(n=127) \text {, and overall }(n=289) \text {. } \\
\ddagger \text { †oes not include recent immigrants and visitors: hepatitis } A(n=38) \text {, malaria }(n=82) \text {, enteric fever }(n=117) \text {, and overall }(n=237) \text {. } \\
\S \text { Not mutually exclusive. }\end{array}$} \\
\hline
\end{tabular}

quarter $(26.2 \%)$ of the healthcare costs of hepatitis A, malaria, and enteric fever in Ontario were accrued in the Peel region, despite this region comprising only $10.3 \%$ of the Ontario population (28).

The average healthcare spending per case-patient $(\$ 6,472)$ was $>17$ times the cost per control $(\$ 375)$, for an attributable additional cost of $\$ 6,098(95 \%$ CI $\$ 5,328-\$ 6,868)$ per case-patient (Table 6). Costs varied by disease and were greatest for enteric fever. Attributable healthcare costs were primarily accrued in inpatient settings, which accounted for $57 \%-70 \%$ of costs across infections (Figure 2), followed by outpatient (22\%-36\%) and ED settings ( $8 \%$ ).

Attributable costs were robust across sensitivity analyses. When restricted to the most relevant healthcare costs, costs were $\approx \$ 1,000$ less for hepatitis A but remained similar for the other infectious diseases (Table 6). A shorter follow-up period of 60 days similarly did not substantially change cost estimates $(\$ 5,859,95 \% \mathrm{CI}$ $\$ 5,126 \$ 6,592$ ). To examine how costs were affected by our decision to include the small number of case-patients with locally acquired hepatitis A and those for which travel exposure was unknown $(\mathrm{n}=10)$, we conducted a post hoc sensitivity analysis excluding these case-patients. The overall attributable cost remained relatively unchanged at $\$ 5,942(95 \%$ CI $\$ 5,247 \$ 6,637)$ per casepatient, and the mean hepatitis A-specific attributable cost was reduced to $\$ 3,710$ (95\% CI 95\% CI $\$ 2,044$ $\$ 5,376$ ) per case-patient.

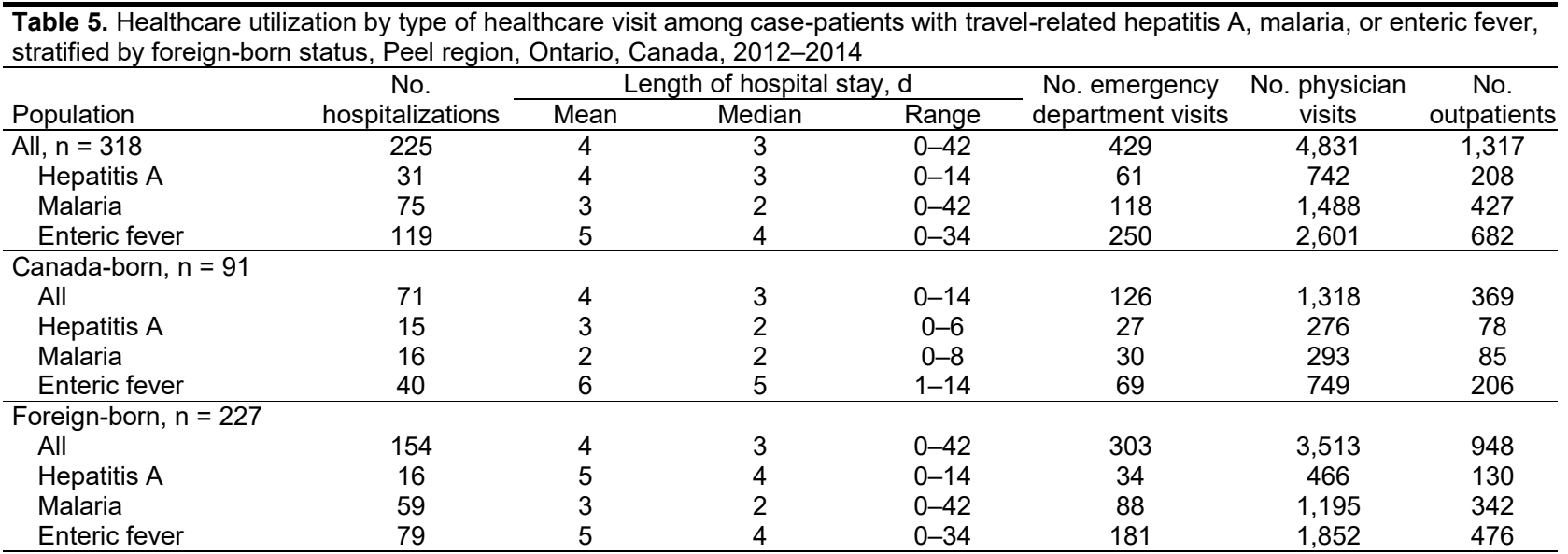


Table 6. Total direct and attributable medical costs of hepatitis A, malaria, and enteric fever, Peel region, Ontario, Canada, 2012-2014*

\begin{tabular}{|c|c|c|c|c|c|c|c|}
\hline \multirow[b]{2}{*}{ Category } & \multicolumn{5}{|c|}{ Peel region } & \multicolumn{2}{|c|}{ Ontario } \\
\hline & $\begin{array}{l}\text { No. case- } \\
\text { patients }\end{array}$ & $\begin{array}{l}\text { Total direct } \\
\text { costs, } \$\end{array}$ & $\begin{array}{l}\text { Cost per case- } \\
\text { patient, \$, mean } \\
\text { (range) }\end{array}$ & $\begin{array}{c}\text { Cost per } \\
\text { control, \$, mean } \\
\text { (range) }\end{array}$ & $\begin{array}{c}\text { Attributable cost } \\
(95 \% \mathrm{Cl}), \$\end{array}$ & $\begin{array}{c}\text { No. } \\
\text { case- } \\
\text { patients }\end{array}$ & $\begin{array}{c}\text { Total direct } \\
\text { costs, } \$ \dagger\end{array}$ \\
\hline Overall 90-d cost & 318 & $2,058,196$ & $6,472(0-59,358)$ & $375(0-42,213)$ & $6,098(5,328-6,868)$ & 1,216 & $7,870,341$ \\
\hline \multicolumn{8}{|l|}{ By disease } \\
\hline Hepatitis A & 55 & 306,707 & $5,576(0-59,358)$ & $560(0-42,213)$ & $5,016(2,414-7,619)$ & 300 & $1,672,944$ \\
\hline Malaria & 122 & 613,488 & $5,029(0-38,556)$ & $471(0-26,598)$ & $4,558(3,557-5,558)$ & 612 & $3,077,497$ \\
\hline Plasmodium vivax & 43 & 230,487 & ND & ND & $4,812(2,675-6,949)$ & ND & ND \\
\hline P. falciparum & 59 & 297,106 & ND & ND & $4,743(3,588-5,898)$ & ND & ND \\
\hline Other & 20 & 85,895 & ND & ND & $3,463(1,397-5,528)$ & ND & ND \\
\hline Enteric fever & 141 & $1,138,002$ & $8,071(0-33,563)$ & $219(0-13,824)$ & $7,852(6,812-8,893)$ & 304 & $2,453,566$ \\
\hline \multicolumn{8}{|c|}{ Restricted to most relevant costs } \\
\hline Hepatitis A & 55 & 233,771 & $4,250(0-23,048)$ & $266(0-10,163)$ & $3,984(2,823-5,145)$ & 300 & $1,275,117$ \\
\hline Malaria & 122 & 611,030 & $5,008(0-37,335)$ & $361(0-17,626)$ & $4,647(3,593-5,701)$ & 612 & $3,065,171$ \\
\hline Enteric fever & 141 & $1,029,311$ & $7,300(0-33,563)$ & $211(0-13,824)$ & $7,089(6,097-8,081)$ & 304 & $2,219,224$ \\
\hline
\end{tabular}

\section{Discussion}

By linking reportable disease surveillance data with health administrative data, this study provides comprehensive, population-based estimates of the medical costs of 3 reportable travel-related infections. Total medical costs were $>\$ 2$ million in the Peel region of Ontario during our 3-year study period; scaled up to the provincial level, we estimated close to $\$ 8$ million in healthcare spending for the 1,216 reported cases in Ontario that occurred over the same period. Attributable 90 -day medical costs ranged from $\$ 4,558$ for malaria to $\$ 7,852$ for enteric fever; these estimates are in line with total per capita health expenditures in Ontario of \$6,584 in 2018 (43), highlighting the substantial resources required for diagnosis and treatment of these infections. Costs were primarily driven by care provided in inpatient settings and represent mostly avoidable healthcare spending, considering that safe and effective medical interventions (e.g., pretravel health consultations, immunization, chemoprophylaxis) are available to prevent infection and clinical disease.

To the best of our knowledge, the comprehensive medical costs of hepatitis A, malaria, and enteric fever have not been estimated elsewhere. In London, England, where rates of reportable travel-related infections are high and reflective of an ethnically diverse population, inpatient costs have been estimated at $£ 1,375(\approx 2015$ Can $\$ 2,300)$ per admission for malaria and $£ 1,976(\approx 2015$ Can $\$ 3,300)$ per admission for typhoid $(25,26)$. These values are comparable, albeit slightly lower than the costs reported in our study, probably because of their restriction to the inpatient setting and lower healthcare costs in the United Kingdom $(38,44)$. In both settings, costs of enteric fever were greater than those of malaria. This difference might be attributable to the poor sensitivity of available microbiological tests for the detection of Salmonella enterica serovars Typhi and Paratyphi, which can lead to repeated testing and delays in receiving appropriate treatment (45). Emerging antimicrobial resistance also contributes to costs. In a retrospective chart review of a large, tertiary care pediatric center in Toronto, only $40 \%$ of isolates were found to be fully susceptible to the drugs typically used to treat enteric fever, and $64 \%$ of patients needed to be recalled to the hospital after positive blood cultures (46). Improving diagnostics for enteric fever is critical to reducing hospitalizations and associated healthcare spending.

Although public health costs related to case and contact management are substantial, they are often excluded because they are not routinely or systematically tracked. Omission of these expenditures can lead to underestimates of disease burden, particularly for infections with hepatitis A virus and S. enterica serovars Typhi and Paratyphi, which require follow-up and resource-intensive postexposure prophylaxis (in cases of hepatitis A) to prevent secondary transmission (47). In 2014, Peel Public Health tracked staff hours spent to manage reported travel-related cases and estimated personnel costs of Can $\$ 3,500$ per case for hepatitis A, Can $\$ 3,300$ per case for enteric fever, and Can $\$ 40$ per case for malaria (M. Varia, unpub. data). These estimates are in line with reports from the United States (US $\$ 3,221$ per hepatitis A case) (48). However, costs can be $>$ US $\$ 40,000$ when including the cost of immunoglobulin and vaccines (49).

Overall, $>70 \%$ of the case-patients in our study were foreign-born, suggesting that more needs to be done to ensure equitable access to these interventions for those who are at greatest risk. Although public funding of pretravel health services for VFR travelers might eliminate cost-related barriers, other factors need to be considered and addressed to effectively reduce disease burden in this population. Qualitative studies have found that social contexts and relationships are mediators of the choices and behaviors of 


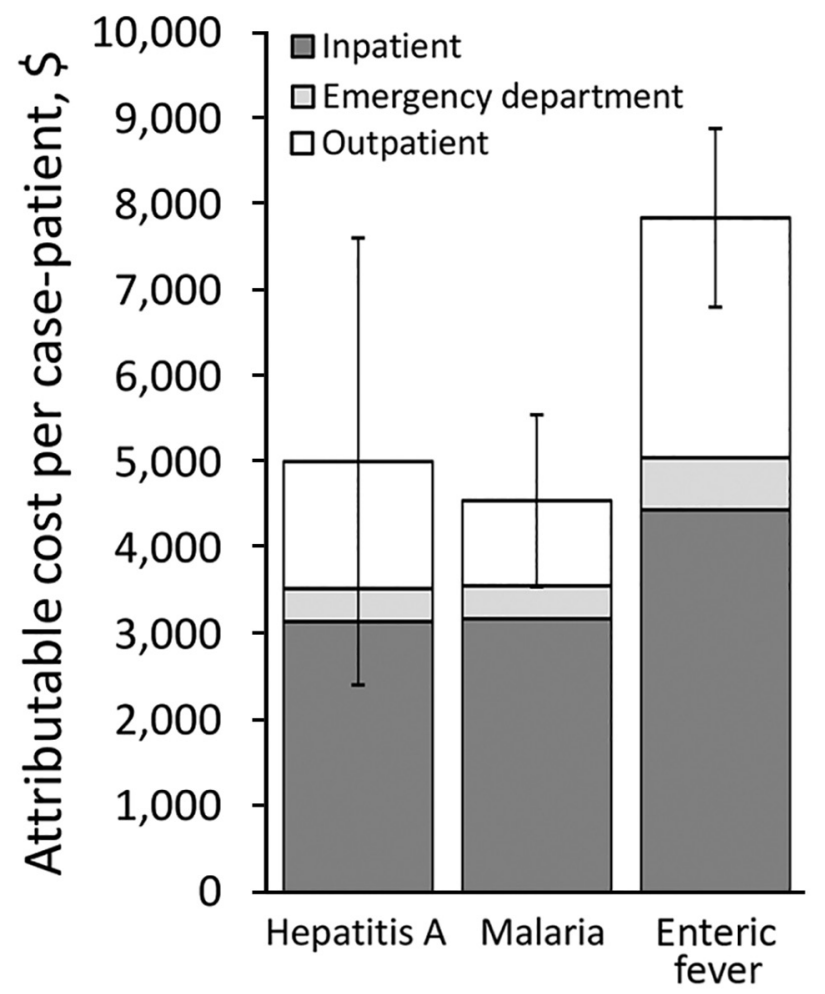

Figure 2. Attributable 90-day healthcare costs in study of direct costs of hepatitis A, malaria, and enteric fever, by disease and healthcare sector, Peel region, Ontario, Canada, 2012-2014. Cost is given in 2015 Canadian dollars. Error bars indicate 95\% Cls.

VFR travelers $(20,22)$. Unlike business or leisure travelers, VFR travelers often stay with family members when visiting and are part of collectivist cultures that place a strong emphasis on acting in the best interest of the family rather than an individual member. These cultural value systems might influence VFR travelers to make decisions to maintain family harmony at the expense of personal health and to go against medical or public health advice to be able to fully participate in cultural activities or rituals that are of value to them (20). They also might need to travel in rushed circumstances to attend to a sick or dying relative. To be effective, public health interventions need to consider the complex social environment in which VFR travelers make decisions. Interventions could include leveraging family in peer-education programs, providing culturally appropriate strategies for making safe food and drink choices that are respectful of hosts and enable travelers to participate in valued rituals, and developing streamlined planning resources for rushed travelers.

Because we took the perspective of the healthcare payer, we were unable to estimate costs incurred by new immigrants not yet eligible for publicly funded healthcare. The financial burden, emotional hardship, and negative health impact of the 3-month waiting period policy have been highlighted in a qualitative study (50). Whether and how new immigrants access care for reportable travel-related infections and subsequent risks for secondary transmission merits further investigation.

Total costs could have also been underestimated if relevant healthcare encounters of case-patients preceded the index date. The costing method we used, though comprehensive, did not include medical costs incurred by community health centers. Because these centers provide care to a small proportion $(1 \%)$ of Ontario's population, their exclusion was not anticipated to appreciably change our results. Our analysis also does not consider costs related to secondary cases or outbreaks, although no locally identifiable outbreaks of hepatitis A or enteric fever occurred in the Peel region during the study period.

For pragmatic reasons, our analysis focused on 3 reportable travel-related infections. The true burden of preventable travel-related infections on the healthcare system is expected to be much larger. Likewise, this analysis does not include healthcare costs for other adverse health events associated with travel, such as events related to concurrent medical conditions, incubating disease events, trauma, and heat- and smog-related illnesses that likely also substantially affect travelers. Although case-patients and controls were comparable by key confounding variables available in the health administrative datasets, they might have differed by other factors that were not measurable with the existing data (e.g., factors that influence healthcare-seeking behaviors and costs), which could have led to an overestimation or underestimation of the attributable cost. Last, we estimated the costs of reportable travel-related infections in a VFR traveler population that was primarily foreign-born and traveling to India and Pakistan. Findings might not be generalizable to other settings with different traveler types and travel patterns.

In conclusion, we found that the attributable medical costs of 3 key reportable travel-related infections were substantial and concentrated in the Peel region of Ontario among immigrants who traveled to visit friends or relatives. Our results could be used to parameterize economic evaluations aimed at determining whether subsidizing or eliminating costs of pretravel health services for high-risk travelers or other policies might be cost-effective. As more and more citizens travel and have links to developing countries through birth or parentage, policy makers must consider equitable strategies that are responsive to the evolving health needs of their populations.

\section{Acknowledgment}

The authors thank Alex Kopp for methodologic support and the staff of Immigration, Refugees, and Citizenship Canada for providing data to ICES, where the analyses were conducted. 
This study was supported by ICES, which is funded by an annual grant from the Ontario Ministry of Health and Long-Term Care. Parts of this material are based on data and/or information compiled and provided by the Canadian Institute for Health Information. However, the analyses, conclusions, opinions, and statements expressed in the material are those of the authors, and not necessarily those of the Canadian Institute for Health Information.

\section{About the Author}

Dr. Savage is an epidemiologist and postdoctoral fellow at Women's College Hospital, Toronto, Ontario, Canada. Her interests are in conducting collaborative, applied public health research focused on improving the health of vulnerable populations, including immigrants, older adults, and women.

\section{References}

1. Boggild AK, Geduld J, Libman M, Yansouni CP, McCarthy AE, Hajek J, et al. Surveillance report of Zika virus among Canadian travellers returning from the Americas. CMAJ. 2017;189:E334-40. http://dx.doi.org/10.1503/cmaj.161241

2. De Serres G, Markowski F, Toth E, Landry M, Auger D, Mercier M, et al. Largest measles epidemic in North America in a decade-Quebec, Canada, 2011: contribution of susceptibility, serendipity, and superspreading events. J Infect Dis. 2013;207: 990-8. http://dx.doi.org/10.1093/infdis/jis923

3. Boggild AK, Geduld J, Libman M, Ward BJ, McCarthy AE, Doyle PW, et al. Travel-acquired infections and illnesses in Canadians: surveillance report from CanTravNet surveillance data, 2009-2011. Open Med. 2014;8:e20-32.

4. Public Health Ontario. Reportable disease trends in Ontario, 2014 technical report. 2016 Apr [cited 2019 Feb 12]. https://www.publichealthontario.ca/-/media/documents/ rdto-2014.pdf?la=en

5. Brophy J, Bui Y, Crockett M, Greenaway C, McCarthy A, Ellia L, et al.; Committee to Advise on Tropical Medicine and Travel. Statement on international travellers who intend to visit friends and relatives. An advisory committee statement (ACS). 2015 Apr [cited 2019 Feb 12]. https://www.canada.ca/content/dam/ phac-aspc/migration/phac-aspc/tmp-pmv/catmat-ccmtmv/assets/ pdfs/friends-amis-eng.pdf

6. Zimmer R. A forgotten vulnerable group: Canadian children visiting relatives in the developing world. CMAJ. 2018;190:E1023. http://dx.doi.org/10.1503/cmaj.70191

7. Lee CS, Gregson DB, Church D, Laupland KB, Eckhardt R, Ross T, et al. Population-based laboratory surveillance of imported malaria in metropolitan Calgary, 2000-2011. PLoS One. 2013;8:e60751. http://dx.doi.org/10.1371/journal.pone.0060751

8. Horn M, Ratansi A, Varia M. Encouraging travellers to take preventive measures against travel-related communicable diseases: a rapid review of the literature. 2013 Jul 12 [cited 2019 Feb 12]. http://www.peelregion.ca/health/library/pdf/travellers-preventivemeasures.pdf

9. Bacaner N, Stauffer B, Boulware DR, Walker PF, Keystone JS. Travel medicine considerations for North American immigrants visiting friends and relatives. JAMA. 2004;291:2856-64. http://dx.doi.org/10.1001/jama.291.23.2856

10. Ericsson CD, Hatz C, Leder K, Tong S, Weld L, Kain KC, et al.; GeoSentinel Surveillance Network. Illness in travelers visiting friends and relatives: a review of the GeoSentinel Surveillance Network. Clin Infect Dis. 2006;43:1185-93. http://dx.doi.org/ $10.1086 / 507893$
11. Bui YG, Trépanier S, Milord F, Blackburn M, Provost S, Gagnon S. Cases of malaria, hepatitis A, and typhoid fever among VFRs, Quebec (Canada). J Travel Med. 2011;18:373-8. http://dx.doi.org/10.1111/j.1708-8305.2011.00556.x

12. Baggett HC, Graham S, Kozarsky PE, Gallagher N, Blumensaadt S, Bateman J, et al. Pretravel health preparation among US residents traveling to India to VFRs: importance of ethnicity in defining VFRs. J Travel Med. 2009;16:112-8. http://dx.doi.org/10.1111/j.1708-8305.2008.00284.x

13. Van Herck K, Castelli F, Zuckerman J, Nothdurft H, Van Damme P, Dahlgren AL, et al. Knowledge, attitudes and practices in travel-related infectious diseases: the European airport survey. J Travel Med. 2004;11:3-8. http://dx.doi.org/10.2310/7060.2004.13609

14. Schlagenhauf P, Weld L, Goorhuis A, Gautret P, Weber R, von Sonnenburg F, et al.; EuroTravNet. Travel-associated infection presenting in Europe (2008-12): an analysis of EuroTravNet longitudinal, surveillance data, and evaluation of the effect of the pre-travel consultation. Lancet Infect Dis. 2015; 15:55-64. http://dx.doi.org/10.1016/S1473-3099(14)71000-X

15. Committee to Advise on Tropical Medicine and Travel. Statement on hepatitis vaccines for travellers. An advisory committee statement (ACS). Can Commun Dis Rep. 2008;34(ACS-2):1-24.

16. Greenaway C, Schofield S, Henteleff A, Plourde P, Geduld J, Abdel-Motagally M, et al. Summary of the statement on international travellers and typhoid by the Committee to Advise on Tropical Medicine and Travel (CATMAT). Can Commun Dis Rep. 2014;40:60-70. http://dx.doi.org/10.14745/ccdr.v40i04a01

17. Boggild A, Brophy J, Charlebois P, Crockett M, Geduld J, Ghesquiere W, et al.; Committee to Advise on Tropical Medicine and Travel. Canadian recommendations for the prevention and treatment of malaria: an advisory committee statement (ACS). 2014 May [cited 2019 Feb 12]. http://publications.gc.ca/pub?id=9.699136\&sl $=0$

18. Ontario Ministry of Health and Long-Term Care. Fact sheet: travel medicine services. Ontario health insurance plan. 1998 [cited 2019 Feb 12]. http://www.health.gov.on.ca/en/pro/programs/ohip/ bulletins $/ 4317 /$ bul4317b.aspx

19. Seale H, Kaur R, Mahimbo A, MacIntyre CR, Zwar N, Smith M, et al. Improving the uptake of pre-travel health advice amongst migrant Australians: exploring the attitudes of primary care providers and migrant community groups. BMC Infect Dis. 2016;16:213. http://dx.doi.org/10.1186/s12879-016-1479-1

20. Savage RD, Rosella LC, Crowcroft NS, Arneja J, de Villa E, Horn M, et al. How can we keep immigrant travelers healthy? Health challenges experienced by Canadian South Asian travelers visiting friends and relatives. Qual Health Res. 2018;28:610-23. http://dx.doi.org/10.1177/1049732317746381

21. Angell SY, Cetron MS. Health disparities among travelers visiting friends and relatives abroad. Ann Intern Med. 2005;142:67-72. http://dx.doi.org/10.7326/0003-4819-142-1-200501040-00013

22. Neave PE, Behrens RH, Jones CO. "You're losing your Ghanaianess": understanding malaria decision-making among Africans visiting friends and relatives in the UK. Malar J. 2014;13:287. http://dx.doi.org/10.1186/1475-2875-13-287

23. Dario Balca. Travel vaccines should be covered by OHIP: Toronto Public Health. CTV News Toronto. 2015 Jul 1 [cited 2019 Feb 12]. https://toronto.ctvnews.ca/travel-vaccines-should-be-covered-byohip-toronto-public-health-1.2449553

24. Harling R, Crook P, Lewthwaite P, Evans M, Schmid ML, Beeching NJ. Burden and cost of imported infections admitted to infectious diseases units in England and Wales in 1998 and 1999. J Infect. 2004;48:139-44. http://dx.doi.org/10.1016/ S0163-4453(03)00080-X

25. Reddy S, Rangaiah J, Addiman S, Wareham D, Wilson P, Sefton A. Epidemiology, antibiotic resistance trends and the cost of 
enteric fever in East London, 2005-2010. Travel Med Infect Dis. 2011;9:206-12. http://dx.doi.org/10.1016/j.tmaid.2011.03.003

26. Rees E, Saavedra-Campos M, Usdin M, Anderson C, Freedman J, de Burgh J, et al. Trend analysis of imported malaria in London; observational study 2000 to 2014. Travel Med Infect Dis. 2017;17:35-42. http://dx.doi.org/10.1016/j.tmaid.2017.04.004

27. Peel Data Centre, Region of Peel. 2011 NHS bulletin: immigration and citizenship. 2013 May [cited 2019 Feb 12]. http://www.peelregion.ca/planning/pde/pdf/Immigration Citizenship_Bulletin.pdf

28. Statistics Canada. Census profiles, 2016 Census. 2018 May 30 [cited 2019 Feb 12]. http://www12.statcan.gc.ca/censusrecensement $/ 2016 / \mathrm{dp}$-pd/prof/index.cfm?Lang=E

29. Government of Ontario. Health Protection and Promotion Act, R.S.O. 1990, c. H.7. 2011.

30. Harron KL, Doidge JC, Knight HE, Gilbert RE, Goldstein H, Cromwell DA, et al. A guide to evaluating linkage quality for the analysis of linked data. Int J Epidemiol. 2017;46:1699-710. http://dx.doi.org/10.1093/ije/dyx177

31. Canadian Institute for Health Information. DAD abstracting manual, 2015-2016 Edition. Ottawa, Canada: The Institute; 2015.

32. Wilkins R. PCCF+ Version 4D User's Guide (Geocodes/PCCF). Automated geographic coding based on the Statistics Canada postal code conversion files, including postal codes to December 2003. Ottawa (Canada): Statistics Canada; 2004.

33. Chiu M, Lebenbaum M, Lam K, Chong N, Azimaee M, Iron K, et al. Describing the linkages of the Immigration, Refugees and Citizenship Canada Permanent Resident data and Vital Statistics Death Registry to Ontario's administrative health database. BMC Med Inform Decis Mak. 2016;16:135. http://dx.doi.org/10.1186/ s12911-016-0375-3

34. Goel R, Beder M. Welcome to Canada ... but don't get sick. CMAJ. 2012;184:E103. http://dx.doi.org/10.1503/cmaj.111095

35. The Johns Hopkins University Bloomberg School of Public Health. The Johns Hopkins ACG® System: excerpt from technical reference guide version 9.0. 2009 Dec [cited 2019 Feb 12]. https://www.healthpartners.com/ucm/groups/public/@hp/@public/ documents/documents/dev_057914.pdf

36. Austin PC, Walraven C. The mortality risk score and the ADG score: two points-based scoring systems for the Johns Hopkins Aggregated Diagnosis Groups to predict mortality in a general adult population cohort in Ontario, Canada. Med Care. 2011;49:940-7. http://dx.doi.org/10.1097/MLR.0b013e318229360e

37. Heymann D. Control of communicable diseases manual. 19th edition. Washington, DC: American Public Health Association; 2008.

38. Wodchis WP, Bushmeneva K, Nikitovic M, McKillop I. Guidelines on person-level costing using administrative databases in Ontario. 2013 May [cited 2019 Feb 12]. http://www.hsprn.ca/uploads/files/ Guidelines_on_PersonLevel_Costing_May_2013.pdf

39. Normand ST, Landrum MB, Guadagnoli E, Ayanian JZ, Ryan TJ, Cleary PD, et al. Validating recommendations for coronary angiography following acute myocardial infarction in the elderly: a matched analysis using propensity scores. J Clin Epidemiol. 2001;54:387-98. http://dx.doi.org/10.1016/ S0895-4356(00)00321-8

40. Austin PC. An introduction to propensity score methods for reducing the effects of confounding in observational studies. Multivariate Behav Res. 2011;46:399-424. http://dx.doi.org/ 10.1080/00273171.2011.568786

41. Rosella LC, Lebenbaum M, Fitzpatrick T, O’Reilly D, Wang J, Booth GL, et al. Impact of diabetes on healthcare costs in a population-based cohort: a cost analysis. Diabet Med. 2016;33:395-403. http://dx.doi.org/10.1111/dme.12858

42. Public Health Ontario. Monthly infectious disease surveillance reports. Data reports 2017 Nov 9 [cited 2019 Feb 12]. https://www.publichealthontario.ca/en/data-and-analysis/ infectious-disease/infectious-diseases-monthly

43. Canadian Institute for Health Information. National health expenditure trends, 1975 to 2018. 2018 Nov [cited 2019 Feb 12]. https://www.cihi.ca/sites/default/files/document/nhex-trendsnarrative-report-2018-en-web.pdf

44. National Health Service England. National tariff payment system 2014/15. 2013 Dec 17 [cited 2019 Feb 12]. https://www.gov.uk/ government/publications/national-tariff-payment-system2014-to-2015

45. Parry CM, Hien TT, Dougan G, White NJ, Farrar JJ. Typhoid fever. N Engl J Med. 2002;347:1770-82. http://dx.doi.org/10.1056/ NEJMra020201

46. Zhou K, Sauve LJ, Richardson SE, Ford-Jones EL, Morris SK. Enteric fever in a multicultural Canadian tertiary care pediatric setting: a 28-year review. J Pediatric Infect Dis Soc. 2017; 6:98-101.

47. Ontario Ministry of Health and Long-Term Care. Infectious diseases protocol, 2016. Ontario Public Health Standards. Toronto: The Ministry; 2016.

48. Epson EE, Cronquist A, Lamba K, Kimura AC, Hassan R, Selvage D, et al. Risk factors for hospitalisation and associated costs among patients with hepatitis A associated with imported pomegranate arils, United States, 2013. Public Health. 2016;136:144-51. http://dx.doi.org/10.1016/j.puhe.2016.03.027

49. Morey RJ, Collier MG, Nelson NP. The financial burden of public health responses to hepatitis A cases among food handlers, 2012-2014. Public Health Rep. 2017;132:443-7. http://dx.doi.org/ $10.1177 / 0033354917710947$

50. Goel R, Bloch G, Caulford P. Waiting for care: effects of Ontario's 3 -month waiting period for OHIP on landed immigrants. Can Fam Physician. 2013;59:e269-75.

Address for correspondence: Rachel D. Savage, Women's College Hospital, 76 Grenville St, Toronto, ON M5S 1B2, Canada; email: rachel.savage@wchospital.ca 